\title{
Cervical Cancer Screening Service Utilization and Associated Factors among Women in the Shabadino District, Southern Ethiopia
}

\author{
Jeylan Kasim, ${ }^{1}$ Abdurehman Kalu, ${ }^{1}$ Bekele Kamara, ${ }^{2}$ and Haileselasie Berhane Alema ${ }^{(1 D}{ }^{3}$ \\ ${ }^{1}$ College of Health Sciences and Referral Hospital, Madda Walabu University, Goba, Ethiopia \\ ${ }^{2}$ Southern Nations Nationalities and People Region Health Bureau, Shabadino District Hospital, Sidama, Ethiopia \\ ${ }^{3}$ Department of Public Health, College of Health Sciences, Aksum University, Axum, Ethiopia \\ Correspondence should be addressed to Haileselasie Berhane Alema; haileselasieb@yahoo.com
}

Received 4 December 2019; Revised 27 April 2020; Accepted 20 May 2020; Published 3 July 2020

Academic Editor: Eleanor Kane

Copyright (c) 2020 Jeylan Kasim et al. This is an open access article distributed under the Creative Commons Attribution License, which permits unrestricted use, distribution, and reproduction in any medium, provided the original work is properly cited.

\begin{abstract}
Background. Cervical cancer is the major cause of morbidity and mortality among women worldwide with an estimated 528,000 new cases and 266,000 deaths annually. In Ethiopia, there are 7095 new cases and 4732 deaths of cervical cancer every year. But cervical cancer screening utilization remains limited. Therefore, the aim of the study was to assess cervical cancer screening utilization and associated factors among women in the Shabadino district, Southern Ethiopia. Methods. A community-based cross-sectional study was conducted in the Shabadino district, Southern Ethiopia, using a structured questionnaire. A systematic random sampling method was used to recruit 536 study participants. The collected data were entered and analyzed using SPSS version 22.0. Bivariate and multivariate logistic regressions were used to assess factors associated with cervical cancer screening utilization at a 95\% level of significance and a $p$ value of less than 0.05 . Results. The study revealed that among 506 women, only $52(10.3 \%)$ have been screened for cervical cancer. Women who are educated (completed primary school and above) $(\mathrm{AOR}=1.9 ; 95 \% \mathrm{CI}=1.18-3.05)$, who have a history of the presence of sexually transmitted diseases $(\mathrm{AOR}=2.6$; $95 \% \mathrm{CI}=1.26-5.23)$, who have multiple sexual partners $(\mathrm{AOR}=4.0 ; 95 \% \mathrm{CI}=1.86-8.66)$, and who knew methods of cervical cancer prevention $(\mathrm{AOR}=4.3 ; 95 \% \mathrm{CI}=1.18-13.05)$ were significantly associated with high cervical cancer screening utilization. Conclusion. The magnitude of cervical cancer screening utilization among women was very low. Educational status, history of multiple sexual partners, history of sexually transmitted diseases, and knowing methods of prevention were significant factors of high cervical cancer screening utilization. Recommendation. It is very crucial to implement an appropriate awareness creation method. Additionally, the STI clinic should be linked to the cervical cancer screening service to increase the knowledge of cervical cancer prevention and the utilization of cervical cancer screening.
\end{abstract}

\section{Background}

Cervical cancer is one of the gravest threats to women's lives. Worldwide, currently, it is estimated that over a million women have cervical cancer. Most of these women have not been diagnosed, nor they have access to treatment that could cure them or prolong their lives [1].

Cervical cancer is the fourth most common cancer in women, and seventh overall, with an estimated 528,000 new cases worldwide. A majority (around $85 \%$ ) of the global burden occurs in the less developed regions. There are about 266,000 deaths from cervical cancer worldwide that accounts for $7.5 \%$ of all female cancer deaths. Almost nine out of ten (87\%) cervical cancer deaths occur in less developed regions [2].

In sub-Saharan Africa, 34.8 new cases of cervical cancer are diagnosed and 22.5 die per 100,000 women annually [3]. In Ethiopia, cervical cancer ranks the second most common type of cancer among women. Every year, 7095 women are diagnosed with cervical cancer, and 4732 die from the disease $[4,5]$. Though there were only a few studies in Ethiopia, the cervical cancer screening service utilization result ranged from $4.8 \%$ to $19.8 \%$ [6-8]. 
According to the American Cancer Society Report, risk factors for cervical cancer include sexual intercourse at an early age, multiple sexual partners, tobacco smoking, longterm oral contraceptive use, low socioeconomic status, immunosuppressive therapy, and micronutrient deficiency [9].

The main strategies of cervical cancer prevention are immunization of human papilloma virus (HPV) vaccine and screening for cervical cancer to detect and remove cancerous lesions. Regular screening and early treatment highly decrease the incidence of cervical cancer. To reduce the incidence and mortality associated with the disease, early screening and treatment as part of targeted interventions is mandatory. So, concrete evidence is crucial for the development of strategies, policy, and planning. But there are very limited studies conducted in the country to assess the utilization of cervical cancer screening. Therefore, this study is aimed at assessing cervical cancer screening service utilization and associated factors among women of 30-49 aged in the Shabadino district, Ethiopia.

\section{Methods}

2.1. Study Settings. This study was conducted in the Shabadino district, Sidama zone, Southern Ethiopia. The district comprises 35 rural kebeles. It is 27 kilometers way from Hawassa, the capital city of the southern region. As shown from the estimated projection of 2007 Central Statistical Agency [10], the district has a total population of 267,487. From this population, 131,068 (49.3\%) are males and $136,419(50.7 \%)$ are females. The district has a total of 54,587 households. There are 45 health facilities in the district which include one district hospital, nine health centers, and 35 health posts. But from health facilities, only one health center offers cervical cancer screening services [11]. The community-based cross-sectional study design was conducted from February to March 2018.

2.2. Study Population. The study population included women whose age ranged from 30 to 49 years in the Shabadino district in the past one year, whereas women with a history of cervical and/or uterus removal and who are positive for cervical cancer were excluded.

2.3. Sample Size Determination. The sample size was determined using a single population proportion formula based on assumptions of $95 \%$ confidence interval, utilization of cervical cancer screening from a previous similar population study, which was 19.8 [6] and with marginal error of $5 \%$.

$$
n=\frac{(Z \alpha / 2)^{2} p(1-p)}{d^{2}} .
$$

The sample size was 244 , and due to the multistage sampling technique, the design effect applied by multiplying with 2 and $10 \%$ of nonresponse rate was added to get the final sample size of 536 .

2.4. Sampling Procedure. Among the total 35 kebeles found in the Shabadino district, 11 kebeles were selected by a simple random sampling technique. Then, 536 households were selected using a systematic random sampling technique and the total sample size was allocated proportionate selected kebele to the size of their households.

Based on the national plan of 2007, the projected estimate of the district comprised 10,568 households in eleven selected kebeles. Therefore, the sampling interval of households in each kebele was determined by dividing the number of households to the allocated sample size of the respective kebeles. The initial household was selected randomly by a lottery method. The subsequent households included in the study were identified by systematic random sampling through house-to-house visit after nominating and adding the sample interval to each household with the pervious number till the total number of sample size was achieved. If there was a household with more than one eligible woman, one woman was randomly selected using the lottery method. Then, the objective of the study was explained by the data collector and consent was asked to participate in the study.

2.5. Data Quality Assurance. The questionnaire was prepared in English and translated to "SidamuAfoo" and retranslated to check its consistency. All were trained for three days on the objective of the study, the contents of the questionnaire, the issues related to the confidentiality of the response, and the right of the respondents. Close supervision was conducted during the process of data collection, and questionnaires were checked for consistency and completeness.

2.6. Data Processing and Analysis. After data collection was completed and questionnaires were edited and coded, the data were entered into a computer and processed by using the Statistical Package for the Social Sciences (SPSS) version 22.0 for further analysis. Descriptive statistics like frequency, percentage, and mean with standard deviation were used to describe the study population in relation to relevant variables. Binary logistic regression analysis with a $95 \%$ confidence interval was used to assess the eligible variable for the multivariable analysis model. And variables that had a significant association with the outcome variable at $p$ value less than 0.2 were entered into the multivariate analysis model. Adjusted odds ratio (AOR) with a 95\% confidence interval (CI) and $p$ value of less than 0.05 were used to identify factors associated with the utilization of cervical cancer screening.

\subsection{Operational Definitions}

2.7.1. Cervical Cancer Screening Utilization. Cervical cancer screening utilization was assessed by asking the respondent's action towards screening for cervical cancer within one year. Those who screened within the past one year were categorized as having utilized, and those who never screened were labeled as having not utilized [12].

2.7.2. Good Knowledge. The scores of the knowledge base item questions were computed, and a score greater than the mean score was considered good knowledge whereas below the mean score is poor knowledge [13]. 
TABLE 1: Sociodemographic characteristics of respondents in the Shabadino district, Sidama Zone, Ethiopia, $2018(n=506)$.

\begin{tabular}{|c|c|c|}
\hline Variables & Frequency & Percentage (\%) \\
\hline \multicolumn{3}{|l|}{ Age group } \\
\hline $30-34$ & 178 & 35.2 \\
\hline $35-39$ & 193 & 38.1 \\
\hline $40-44$ & 85 & 16.8 \\
\hline $45-49$ & 50 & 9.9 \\
\hline \multicolumn{3}{|l|}{ Religion } \\
\hline Orthodox & 45 & 8.9 \\
\hline Protestant & 403 & 79.6 \\
\hline Muslim & 54 & 10.7 \\
\hline Catholic & 4 & 0.8 \\
\hline \multicolumn{3}{|l|}{ Ethnicity } \\
\hline Sidama & 447 & 88.3 \\
\hline Amara & 42 & 8.3 \\
\hline Silite & 7 & 1.4 \\
\hline Wolayita & 10 & 2 \\
\hline \multicolumn{3}{|l|}{ Marital status } \\
\hline Single & 5 & 1 \\
\hline Married & 468 & 92.5 \\
\hline Divorced & 15 & 3 \\
\hline Widowed & 18 & 3.5 \\
\hline \multicolumn{3}{|l|}{ Educational status } \\
\hline Non-formal education & 185 & 36.6 \\
\hline Primary education & 239 & 47.2 \\
\hline Secondary school & 64 & 12.6 \\
\hline College and above & 18 & 3.6 \\
\hline \multicolumn{3}{|l|}{ Occupation } \\
\hline Housewife & 421 & 83.2 \\
\hline Self-employed & 70 & 13.8 \\
\hline Government employee & 15 & 3 \\
\hline \multicolumn{3}{|l|}{ Household monthly income } \\
\hline$<900$ & 146 & 28.9 \\
\hline $901-1600$ & 258 & 51 \\
\hline $1601-2700$ & 83 & 16.4 \\
\hline$>2700$ & 19 & 3.7 \\
\hline
\end{tabular}

\section{Results}

3.1. Sociodemographic Characteristics of Respondents. From a total of 536 selected eligible women, 506 have participated in the study with the response rate of $94 \%$. The mean age of participants was $37( \pm 5.3 \mathrm{SD})$ years (Table 1$)$.

3.2. Sexual and Reproductive Characteristics of Respondents. Almost all $(503,99.4 \%)$ of the participants had sexual intercourse, and among them, 430 (85\%) had their first sexual intercourse at the age of 18 and above (Table 2).

3.3. Knowledge and Prevalence of Cervical Cancer Screening Utilization. From the study participants, 321 (63.4\%) had heard about cervical cancer. Of those who had heard about
TABLE 2: Sexual and reproductive characteristics of respondents in the Shabadino district, Sidama Zone, Ethiopia, $2018(n=506)$.

\begin{tabular}{lcc}
\hline Variables & Frequency & Percentage (\%) \\
\hline Used COC pills & & \\
$\quad$ No & 327 & 64.6 \\
$\quad$ Yes & 179 & 35.4 \\
Had history of STI & & \\
$\quad$ No & 387 & 76.5 \\
$\quad$ Yes & 119 & 23.5 \\
HIV test & & \\
$\quad$ No & 126 & 24.9 \\
$\quad$ Yes & 380 & 75.1 \\
Serostatus & & \\
$\quad$ Negative & 392 & 77.5 \\
Positive & 114 & 22.5 \\
Had given birth & & \\
$\quad$ No & 8 & 1.6 \\
$\quad$ Yes & 498 & 98.4 \\
Age at first sexual intercourse & & \\
$\quad<18$ & 89 & 17.6 \\
$\quad \geq 18$ & 417 & \\
Multiple sexual partners & & \\
No & 446.1 \\
$\quad$ Yes & 60 & \\
\hline
\end{tabular}

cervical cancer, 293 (58\%) knew about its risk factors, 215 (42.5\%) knew about its symptoms, and 150 (29.5\%) knew about its methods of prevention.

Concerning their source of information about cervical cancer, mass media was the most common source of information preceding by health workers and health extension workers. Two hundred two (39.9\%) of the respondents have good knowledge, and $304(60.1 \%)$ have poor knowledge about cervical cancer prevention.

The prevalence of cervical cancer screening utilization was found to be $52(10.3 \%)$.

3.4. Factors Associated with Cervical Cancer Screening Utilization. On bivariate analysis, factors found to be significantly associated with utilization of cervical cancer screening service were educational status, household monthly income, hearing about cervical cancer, history of the sexually transmitted disease, HIV test, multiple sexual partners, knowing the causes of cervical cancer, and knowing the prevention methods of cervical cancer.

After controlling for confounders using the multivariate analysis model, educational status, history of sexually transmitted disease, and multiple sexual partners were significantly associated with the cervical cancer screening service utilization.

This study revealed that the educational level has a significant association with the cervical cancer screening utilization; women who attended primary education and above were about 2 times more likely to utilize cervical cancer 
TABLE 3: Bivariate and multivariate analyses of factors associated with cervical cancer screening utilization among women in the Shabadino district, Sidama Zone, Ethiopia, 2018.

\begin{tabular}{|c|c|c|c|c|c|}
\hline \multirow[t]{2}{*}{ Variables } & \multicolumn{2}{|c|}{$\begin{array}{l}\text { Cervical cancer } \\
\text { screening } \\
\text { utilization }\end{array}$} & \multirow[t]{2}{*}{ COR $(95 \%$ CI $)$} & \multirow[t]{2}{*}{ AOR (95\% CI) } & \multirow[t]{2}{*}{$p$ value } \\
\hline & Yes & No & & & \\
\hline \multicolumn{6}{|l|}{ Age group } \\
\hline $30-34$ & 17 & 160 & 1 & & \\
\hline $35-39$ & 22 & 171 & $1.14(0.62-2.36)$ & & \\
\hline $40-44$ & 7 & 77 & $0.92(0.34-2.15)$ & & \\
\hline $45-49$ & 6 & 46 & $0.77(0.46-3.29)$ & & \\
\hline \multicolumn{6}{|l|}{ Educational status } \\
\hline Non-formal education & 9 & 191 & 1 & 1 & \\
\hline Primary education & 20 & 212 & $2.00(0.89-4.50)$ & & \\
\hline Secondary school & 19 & 42 & $9.60(4.06-22.70)$ & $1.89(1.18-3.05)^{*}$ & 0.009 \\
\hline College and above & 4 & 9 & $9.43(2.44-36.54)$ & & \\
\hline \multicolumn{6}{|l|}{ Occupation } \\
\hline House-wife & 38 & 383 & 1 & & \\
\hline Self-employed & 9 & 62 & $1.46(0.67-3.17)$ & & \\
\hline Governmental employee & 5 & 9 & $5.6(12)$ & & \\
\hline \multicolumn{6}{|l|}{ Had history of STI } \\
\hline No & 27 & 360 & 1 & 1 & \\
\hline Yes & 25 & 94 & $3.55(1.97-6.39)$ & $2.57(1.26-5.23)^{*}$ & 0.021 \\
\hline \multicolumn{6}{|l|}{ Age at first sexual intercourse } \\
\hline$<18$ & 27 & 621 & & & \\
\hline$\geq 18$ & 25 & 392 & $0.15(0.08-0.27)$ & & \\
\hline \multicolumn{6}{|c|}{ Have history of multiple sexual partners } \\
\hline No & 414 & 32 & 1 & 1 & \\
\hline Yes & 40 & 20 & $6.5(3.39-12.34)$ & $4.01(1.86-8.66)^{*}$ & 0.001 \\
\hline \multicolumn{6}{|c|}{ Knew the prevention methods of cervical cancer } \\
\hline No & 341 & 15 & 1 & 1 & \\
\hline Yes & 113 & 37 & $7.4(3.94-14.07)$ & $4.34(1.18-3.05)^{*}$ & 0.001 \\
\hline
\end{tabular}

*Statistically associated with cervical cancer screening utilization at $p$ value $<0.05$.

screening service than those who had never attended any formal education $(\mathrm{AOR}=1.89 ; 95 \% \mathrm{CI}=1.18-3.05, p=0.009)$.

Women who have a history of sexually transmitted disease were about 2.6 times more likely to utilize cervical cancer screening when compared to those who have no history of sexually transmitted disease $(\mathrm{AOR}=2.57 ; 95 \% \mathrm{CI}=1.26-5.23)$.

Women who have a history of multiple sexual partners were 4 times more likely to utilize cervical cancer screening when compared to those who have a single partner ( $\mathrm{AOR}=4.01 ; 95 \% \mathrm{CI}=1.86-8.66)$.

Regarding the knowledge status, women who knew that cervical cancer is a preventable disease were 4.3 times more likely to utilize cervical cancer screening service than those who do not know that cervical cancer is a preventable disease $(\mathrm{AOR}=4.34 ; 95 \% \mathrm{CI}=1.18-13.05)$ (Table 3$)$.

\section{Discussion}

This community-based study was conducted to assess the level of cervical cancer screening service utilization and its associated factors among women in the Shabadino district, Sidama Zone, Southern Ethiopia.

This study revealed that only $52(10.3 \%)$ of respondents had screened for cervical cancer. This result was low compared to studies done in different parts of Ethiopia which were $15.5 \%, 16.5 \%, 22 \%$, and $25 \%[14,15,12,16]$. The reason behind this is because the study populations are living in remote areas where they lack access to education and lack women empowerment. But the findings are somehow slightly higher from studies in Nigeria and Arba Minch town, Ethiopia, which were $8.0 \%$ [17] and 5.8\% [7], respectively. This could be explained by the study period difference. Those studies were done some years back where awareness and availability of the service of cervical cancer screening were low.

The factors associated with the utilization of cervical cancer screening were educational status of the woman, presence of sexually transmitted diseases, presence of multiple partners, and knowledge of risk and prevention methods of cervical cancer. 
Educational status was significantly associated with the utilization of cervical cancer screening. Women who attended primary school and above were 2 times more likely to utilize cervical cancer screening service compared to those who did not attend any formal education. This finding was consistent with the study done in Addis Ababa, Ethiopia [18], and Ghana [19]. Education can increase women's access to information from different sources. This could be due to the fact that educated women have better awareness of the benefit of cervical cancer screening service utilization.

The presence of sexually transmitted diseases was significantly associated with cervical cancer screening service utilization. Women who have a history of sexually transmitted diseases were 2.6 times more likely to be screened for cervical cancer than those who have no history of sexually transmitted diseases. This result is consistent with the study findings in different parts of Ethiopia $[6,20,15]$.

Having multiple sexual partners was identified as a risk factor for cervical cancer screening utilization. Our study revealed that women who have a history of multiple sexual partners were 4 times more likely to utilize cervical cancer screening compared to those who have a single partner. The result is supported by studies done in Ethiopia and Malawi $[6,14,21]$. This is because women with multiple sexual partners may have a high risk of contracting sexually transmitted diseases (STDs). Once women develop symptoms for any sexually transmitted diseases, there is an increased chance of seeking medical care.

Knowledge on prevention methods of cervical cancer was found to be significantly associated with cervical cancer screening utilization. This study also showed that women with good knowledge of prevention methods of cervical cancer have a higher chance of cervical cancer screening utilization compared to those with poor knowledge. This result was similar to studies conducted in Hossana, Addis Ababa (Ethiopia), and Nigeria $[12,22,23]$. This is because women with good knowledge of prevention methods of cervical cancer had increased health care-seeking behavior. This makes them have a regular checkup for cervical cancer and other diseases to monitor their health status.

\section{Limitation}

This study involved a sensitive matter that may be subjected to social desirability bias. Meanwhile, the procedure to screen for cervical cancer and precervical lesion was similar for the women. The study participants may face difficulty to differentiate the type they are screened for.

\section{Conclusion}

The study revealed that the magnitude of cervical cancer screening service utilization among women was very low. Educational status, history of multiple sexual partners, presence of sexually transmitted diseases (STDs), and knowledge about prevention methods were significant factors associated with cervical cancer screening service utilization.

\author{
Abbreviations \\ AOR: Adjusted odds ratio \\ COR: Crude odds ratio \\ CCPC: Cervical Cancer Prevention and Control \\ CIN: $\quad$ Cervical intraepithelial neoplasia \\ ETB: Ethiopian birr \\ FDRE: Federal Democratic Republic of Ethiopia \\ HICs: High-income countries \\ HPV: Human papilloma virus \\ IARC: International Agency for Research on Cancer \\ LMICs: Low- and middle-income countries \\ NCCPP: National Cervical Cancer Prevention Program \\ SPSS: $\quad$ Statistical Package for the Social Sciences \\ STI: $\quad$ Sexually transmitted infection \\ VIA: Visual inspection with acetic acid \\ VILI: Visual inspection with Lugol's iodine \\ WHO: World Health Organization.
}

\section{Data Availability}

All the data supporting the findings is contained within the manuscript.

\section{Additional Points}

Recommendation. It is very crucial to implement appropriate awareness creation methods. All women in the STI clinic should be linked to screen for cervical cancer to increase the knowledge of cervical cancer prevention and the utilization of cervical cancer screening. The use of opportunities to provide health education about cervical cancer to women during antenatal care follow-up, during family planning service, and at STI clinic is vital.

\section{Ethical Approval}

Ethical clearance was obtained from the IRB of Madda Walabu University, Goba Referral Hospital. An official letter of permission was also obtained from the respective administrative district office.

\section{Consent}

Each respondent provided written and verbal consent before data collection has been carried out. Names of the respondents were not used to ensure anonymity and confidentiality.

\section{Conflicts of Interest}

The authors declare that they have no competing interests.

\section{Authors' Contributions}

The contributions of the authors involved in this study are as follows: JK and AK: conceived and designed the study; JK, $\mathrm{AK}, \mathrm{BK}$, and $\mathrm{HB}$ : analyzed the data; and $\mathrm{JK}, \mathrm{AK}, \mathrm{BK}$, and HB: prepared the manuscript. All authors read and approved the final manuscript before submission for publication. 


\section{Acknowledgments}

Our especial gratitude goes to Southern Nations Nationalities and Peoples Regional Health Bureau and health institutions for their kind cooperation and continuous support. Finally, we would like to extend our gratitude to the study participants, data collectors and supervisors.

\section{References}

[1] WHO, "WHO. Comprehensive cervical cancer control," in $A$ guide to essential practice, pp. 9-38, WHO Library Cataloguing-in-publication data, 2014.

[2] World Health Organization, Cervical cancer, Cancer Fact sheets-GLOBOCAN, 2012.

[3] (IARC) IAfRoC, Cancer incidence and mortality worldwide, IARC's online database, . GLOBOCAN, 2013.

[4] Health EFMo, National Cancer Control Plan 2016-2020, Addis Ababa, Ethiopia, 2016.

[5] Cancer ICoHa, Human papilloma virus and related cancers, Fact Sheet, 2017.

[6] H. Bayu, Y. Berhe, A. Mulat, and A. Alemu, "Cervical Cancer Screening Service Uptake and Associated Factors among Age Eligible Women in Mekelle Zone, Northern Ethiopia, 2015: A Community Based Study Using Health Belief Model," PLoS ONE, vol. 11, no. 3, p. e0149908, 2016.

[7] Z. Gebru and M. Gerbaba, "Utilization of cervical carcinoma screening service and associated factors among currently married women in Arba Minch town, Southern Ethiopia," Journal of Women's Health Care, vol. 5, no. 1, 2016.

[8] R. Ndejjo, T. Mukama, A. Musabyimana, and D. Musoke, "Uptake of cervical cancer screening and associated factors among women in rural Uganda: A Cross Sectional Study," PLoS ONE, vol. 11, no. 2, p. e0149696, 2016.

[9] American Association for Cancer Research, "Global burden of cancer in women, current status, trends, and interventions," Cancer Epidemiol Biomarkers Prevention, vol. 26, no. 4, pp. 444-457, 2017.

[10] C. S. Authority, Population and housing census of Ethiopia, administrative report 2012, 2007.

[11] Development SdFaE, Socio-economic profile 2012-2014, 2015.

[12] T. Nigussie, B. Admassu, and A. Nigussie, "Cervical cancer screening service utilization and associated factors among age-eligible women in Jimma town using health belief model, South West Ethiopia," BMC Women's Health, vol. 19, no. 1, 2019.

[13] M. T. Segni, H. F. Demissie, D. M. Tadesse, R. Amdemichael, Y. T. Mamo, and A. Worku, "Level of knowledge and associated factor toward cervical cancer among women age (21-64) years visiting health facilities in Gulele sub-city Addis Ababa Ethiopia," Journal of the Pancreas, vol. 18, no. 1, pp. 44-48, 2016.

[14] E. W. Tadesse Tekle, B. Nega, W. P. Kumma, and M. M. Koyira, Knowledge, attitude and practice towards cervical cancer screening among women and associated factors in hospitals of Wolaita zone, Southern Ethiopia, DovePress, 2020.

[15] T. Hailemariam, B. Yohannes, H. Aschenaki, E. Mamaye, G. Orkaido, and M. Seta, "Prevalence of cervical cancer and associated risk factors among women attending cervical cancer screening and diagnosis center at Yirgalem General Hospital,
Southern Ethiopia," Journal of Cancer Science and Theray, vol. 9, no. 11, 2017.

[16] S. Getachew, E. Getachew, M. Gizaw, W. Ayele, A. Addissie, and E. J. Kantelhardt, "Cervical cancer screening knowledge and barriers among women in Addis Ababa, Ethiopia," PLoS One, vol. 14, no. 5, p. e0216522, 2019.

[17] A. Idowu, S. A. Olowookere, A. T. Fagbemi, and O. A. Ogunlaja, "Determinants of Cervical Cancer Screening Uptake among Women in Ilorin, North Central Nigeria: A Community-Based Study," Journal of Cancer Epidemiology, vol. 2016, 8 pages, 2016.

[18] N. Bekele, Willingness and acceptability of cervical cancer screening among women living with HIVIAIDS in selected public health institution of Addis Ababa Ethiopia, unplished article, 2014.

[19] K. Mercy, Factors affecting the utilisation of cervical cancer screening among women attending health services in the Kumasi Metropolis of Ghana, unpublished, 2017.

[20] F. Getahun, F. Mazengia, M. Abuhay, and Z. Birhanu, "Comprehensive knowledge about cervical cancer is low among women in Northwest Ethiopia," BMC Cancer, vol. 13, no. 1, 2013.

[21] M. S. Chosamata, S. A. Hong, and S. Tiraphat, "Determinants of cervical cancer screening utilization among women aged 3045 years in Blantyre district, Malawi," Journal of Public Health and Development, vol. 13, no. 3, 2015.

[22] N. A. Murugi, Determinants of cervical cancer screening uptake among women in Embu County, Kenya, unpublished, 2014.

[23] S. Shiferaw, Knowledge about cervical cancer and barriers towards cervical cancer screening among HIV positive women, in health centers of Addis Ababa, Ethiopia, unpublished, 2016.

[24] Y. H. Aweke, S. Y. Ayanto, and T. L. Ersado, "Knowledge, attitude and practice for cervical cancer prevention and control among women of childbearing age in Hossana Town, Hadiya zone, Southern Ethiopia: community-based cross-sectional study," PLoS ONE, vol. 12, no. 7, p. e0181415, 2017. 